\title{
Selective 6-O-Debenzylation of Mono- and Disaccharide Derivatives Using $\mathrm{ZnCl}_{2}-\mathrm{Ac}_{2} \mathrm{O}-\mathrm{HOAc}$
}

\author{
Guangbin Yang, Xianglan Ding, and Fanzuo Kong* \\ Research Center for Eco-Environmental Sciences, Academia Sinica, P.O.Box 2871, \\ Beijing 100085, P. R. China
}

\begin{abstract}
Freshly fused $\mathrm{ZnCl}_{2}$ in $\mathrm{Ac}_{2} \mathrm{O}$ /HOAc at room temperature has been used for 6-0debenzylation of mono- and disaccharide derivatives giving yields more than $80 \%$. Notably, allyl, acetyl, benzoyl, tosyl, thiono groups are unaffected under the designated conditions. (C) 1997 Elsevier Science Ltd.
\end{abstract}

Many important bioactive natural products contain $1 \rightarrow 6$ glycosidic linkages ${ }^{1-6}$. Selective $6-0-$ debenzylation of methyl glycoside derivatives is a very useful procedure for the synthesis of $1 \rightarrow 6$ linked oligosaccharides since it can substantially simplify the reaction pathway. Although the acetolysis of benzyl ethers initialized by Lewis acid or proton is well known, for example, by $\mathrm{FeCl}_{3} / \mathrm{Ac}_{2} \mathrm{O}^{7}, \mathrm{ZnI}_{2} / \mathrm{Ac}_{2} \mathrm{O}^{8}$, and $\mathrm{H}_{2} \mathrm{SO}_{4} / \mathrm{Ac}_{2} \mathrm{O}^{9}$, the selectivity and yield are not satisfactory. The direct 6-O-debenzylation using trimethylsilyl trifluoromethanesulfonate/acetic anhydride ( $\left.\mathrm{TMSOTf} / \mathrm{Ac}_{2} \mathrm{O}\right)^{10}$ at $-40{ }^{\circ} \mathrm{C}$ has been shown to be temperature dependent, and secondary benzyl ether linkages can also be cleaved as the temperature is raised. Iodotrimethylsilane is another catalyst for direct 6-O-debenzylation ${ }^{11}$ giving moderate to good yield, but the secondary benzyl groups can also be cleaved as the reaction time is long. In our recent studies, we found that $\mathrm{ZnCl}_{2}$ (7.5-10eq)- $\mathrm{Ac}_{2} \mathrm{O}-\mathrm{HOAc}$ was a highly efficient reagent for 6-O-debenzylation at room temperature. Compounds of diverse structure including methyl $\alpha$ - and $\beta$-D-glucopyranoside, methyl $\alpha$-D-galacto, manno, talopyranoside and methyl $1 \rightarrow 6$ linked disaccharide were tried. Notably, allyl, äcetyl, benzoyl, tosyl, thiono groups all survived under the reaction conditions, no anomerization and glycosidic bond breaking were observed (see Table I for results). Also, 6-O-debenzylation of monosaccharides having one free 
hydroxy group (see 2 and 4 ) was very successfully performed giving the products with two potential hydroxy groups capable of building branched oligosaccharides. It was also found that a large excess of freshly fused $\mathrm{ZnCl}_{2}$ (20eq) and a long reaction time (4h) would simultaneously cleave the methyl glycosidic bond while the secondary benzyl groups were still not changed (see Table I for results). The typical procedure for selective 6-O-debenzylation is as follows: To a solution of 6-O-benzylated sugar (50-200mg) in $1 \mathrm{ml} \mathrm{Ac}_{2} \mathrm{O} / \mathrm{HOAc}$

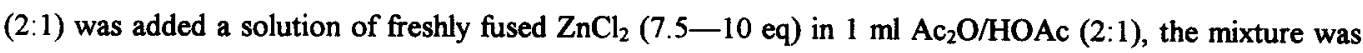
stirred at room temperature for $2 \mathrm{~h}$, TLC ( $3: 1$ petroleum ether-ethyl acetate) indicated that the reaction was complete. Water was added, and the mixture was extracted with dichloromethane three times, washed with saturated sodium carbonate, then water, dried with $\mathrm{Na}_{2} \mathrm{SO}_{4}$, and concentrated to give a syrup. Purification of the syrup by column chromatography (3:1 petroleum ether-ethyl acetate) yielded the pure product (yield $>80 \%$ ). The structures of most of the starting materials and products were simple, among which known compounds $1,1^{12}, 2^{13}, 3,3^{\prime}, 3^{\prime 14}, 5,5^{15}, 5^{\prime 16}, 7^{17}, 9,9^{18}$, and $10,10^{19}$ were identified by comparison of their physical data to the reported values in literature, while new compounds were characterized by optical rotations, elemental analyses, and ${ }^{1} \mathrm{H}$ NMR spectrometry ${ }^{20}$. Compared to the starting materials, the products gave ${ }^{1} \mathrm{H}$ NMR spectra showing a downfield chemical shift of H-6 and upfield shift of the acetyl methyl signal. In summary here we report a very effective and facile method for selective 6-O-debenzylation and this method can be used in the synthesis of oligosaccharides.

Table I Results for selective 6-O-debenzylation using $\mathrm{ZnCl}_{2}-\mathrm{Ac}_{2} \mathrm{O}-\mathrm{HOAc}$

\begin{tabular}{|l|c|c|c|c|c|c|c|c|c|c|}
\hline Compound & $\mathbf{1}$ & $\mathbf{2}$ & $\mathbf{3}$ & $\mathbf{4}$ & $\mathbf{5}$ & $\mathbf{6}$ & $\mathbf{7}$ & $\mathbf{8}$ & $\mathbf{9}$ & $\mathbf{1 0}$ \\
\hline $\mathrm{ZnCl}_{2}$ (eq) & 7.5 & 10 & 7.5 & 10 & 7.5 & 10 & 10 & 10 & 7.5 & 10 \\
\hline Product & $\mathbf{1}$ & $\mathbf{2}$ & $\mathbf{3}$ & $\mathbf{4}$ & $\mathbf{5}$ & $\mathbf{6}$ & $\mathbf{7}$ & $\mathbf{8}$ & $\mathbf{9}$ & $\mathbf{1 0}$ \\
\hline Yield & $85 \%$ & $90 \%$ & $80 \%$ & $86 \%$ & $\mathbf{8 2} \%$ & $\mathbf{8 8 \%}$ & $92 \%$ & $94 \%$ & $94 \%$ & $81 \%$ \\
\hline $\mathrm{ZnCl}_{2}$ (eq) & & 20 & 20 & & 20 & & & 20 & & \\
\hline Product & & $\mathbf{2}$ & $\mathbf{3}$ & & $\mathbf{5}$ & & & $\mathbf{8}$ & & \\
\hline Yield & $86 \%$ & $\begin{array}{l}90 \% \\
\alpha: \beta 4: 1\end{array}$ & & $\begin{array}{l}87 \% \\
\alpha: \beta 6: 1\end{array}$ & & & $92 \%$ & & \\
\hline
\end{tabular}




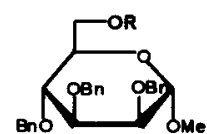

$1,1^{\prime}$

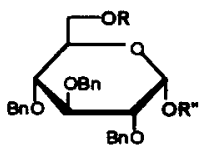

$5,5^{\prime}$

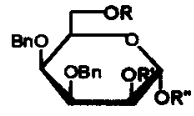

2,2,2"

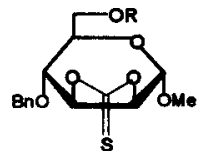

$6,6^{\prime}$

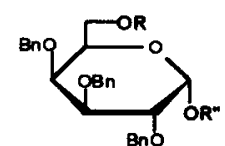

3,3'

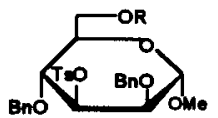

$7,7^{\prime}$

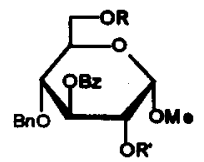

$4,4^{\prime}$

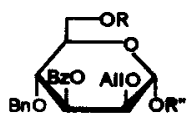

$8,8,8^{\prime \prime}$

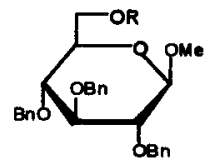

$9,9^{\prime}$

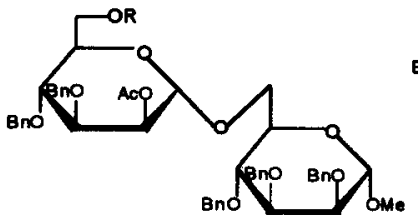

$10,10^{\prime}$

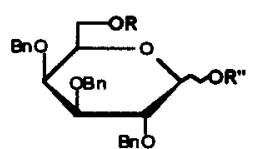

3"

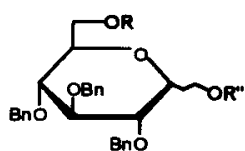

5"

$$
\begin{aligned}
& 1-10 \quad R=B n \quad R^{\prime}=H \quad R^{\prime \prime}=M e \\
& 1^{\prime}-10^{\prime} \quad R=A c \quad R=A c \quad R^{\prime \prime}=M e \\
& 2^{\prime \prime}, 3^{\prime \prime}, 5^{\prime \prime}, 8^{\prime \prime} \quad R=A C \quad R^{\prime}=A C \quad R^{\prime \prime}=A C
\end{aligned}
$$

\section{ACKNOWLEDGEMENT}

Project 29672049 was supported by The National Natural Science Foundation of China.

\section{REFERENCES AND NOTES}

1. Staaf, M.; Widmalm, G.; Yang, Z. and Huffunen, E. Carbohydr. Res., 1996, 291, 155.

2. Jain, R.K. and Matta, K.L. Carbohydr. Res., 1996, 282, 101.

3. Riccio, R.; Kinnel, R.B.; Biofulco, G. and Scheuer, P.J. Tetrahedron lett., 1996, 37, 1979.

4. Nakahara, Y.; Shibayama, S.; Nakahara, Y. and Ogawa, T. Carbohydr. Res., 1996, 280, 67.

5. Nicolaou, K.C.; Winssinger, N.; Pastor, J. and Deroose, F. J. Am. Chem. Soc., 1997, 119, 449.

6. Wyss, D.F.; Choi, J.S. and Wagner, G. Science, 1995, 269, 1273.

7. Ganem, B. and Small, V.R.Jr. J. Org. Chem., 1974, 39, 3728.

8. Edith, S. and Burton, G. J. Chem. Res. Synop., 1990, 8, 248.

9. Sakai, J.I.; Takeda, T. and Ogihara, Y. Carbohydr. Res., 1981, 95, 125. 
10. Angibeaud, P. and Utille, J.P. Synthesis, 1991, 737.

11. Klemer, A.; Bieber, M. and Wilburs, H. Liebigs Ann. Chem., 1983,1416

12. Shah, R.; Baptista, J. and Krepinsky, J.J. J. Carbohydr. Chem.,. 1987, 6(4), 645.

13. Liu, J.; Kong, F. and Cao, L. Carbohydr. Res., 1993, 240, 295.

14. Nashed, M.A. and Anderson, L. Carbohydr. Res., 1976, 51(1), 65.

15. Koto, S.; Morishima, N. and Zen, S. Bull. Chem. Soc. Jpn., 1982, 55(4), 1092.

16. Frechet, J.M. and Schuerch, C. J. Am. Chem. Soc., 1972, 94(2), 604.

17. Kong, F. and Su, B. Carbohydr. Res., 1985, 142(1), 152.

18. Koto, S.; Hamada, Y. and Zen, S. Chem. Lett., 1975, 6, 587.

19. Zhu, X.; Ding, P. and Cai, M. Tetrahedron:Asym., 1996, 7(10), 2833.

20. All new compounds gave satisfactory elemental analysis results. Selected ${ }^{1} \mathrm{H} \mathrm{NMR}\left(\mathrm{CDCl}_{3}, \mathrm{Me}_{4} \mathrm{Si}\right.$ as internal standard) data are as follows: 2' $7.36-7.24(\mathrm{~m}, 10 \mathrm{H}, \mathrm{Ph}-H), 5.34\left(\mathrm{~d}, 1 \mathrm{H}, J_{2,3} 1.7 \mathrm{~Hz}, \mathrm{H}-2\right), 4.94$, 4.75( $\left.\mathrm{AB}_{\mathrm{q}}, 2 \mathrm{H},{ }^{2} \mathrm{~J} 11.7 \mathrm{~Hz}, \mathrm{PhCH}_{2}\right), 4.80(\mathrm{~s}, 1 \mathrm{H}, \mathrm{H}-1), 4.75,4.67\left(\mathrm{AB}_{\mathrm{q}}, 2 \mathrm{H},{ }^{2} \mathrm{~J} 11.7 \mathrm{~Hz}, \mathrm{PhCH}_{2}\right), 4.40-4.18(\mathrm{~m}$, 2H, H-6), 3.92-3.78(m, 3H, H-3,4,5), 3.32(s, 3H OCH 3$), 2.10,2.00\left(2 \mathrm{~s}, 6 \mathrm{H}, 2 \mathrm{COCH}_{3}\right) .2 " .7 .38-7.24(\mathrm{~m}$, $10 \mathrm{H}, \mathrm{Ph}-H), 6.18\left(\mathrm{~d}, 1 \mathrm{H}, J_{1,2} 0.9 \mathrm{~Hz}, \mathrm{H}-1\right), 5.30\left(\mathrm{dd}, 1 \mathrm{H}, J_{2,3} 1.8 \mathrm{~Hz}, \mathrm{H}-2\right), 4.92,4.69\left(\mathrm{AB}_{\mathbf{q}}, 2 \mathrm{H},{ }^{2} \mathrm{~J} 12.0 \mathrm{~Hz}\right.$, $\left.\mathrm{PhCH}_{2}\right), 4.76,4.56\left(\mathrm{AB}_{\mathrm{q}}, 2 \mathrm{H},{ }^{2} \mathrm{~J} 12.0 \mathrm{~Hz}, \mathrm{PhCH}_{2}\right), 4.32-3.32(\mathrm{~m}, 3 \mathrm{H}, \mathrm{H}-3,4,5), 2.10,2.08,2.00(3 \mathrm{~s}, 9 \mathrm{H}$, $\left.3 \mathrm{COCH}_{3}\right)$. 4. 8.04(d, $2 \mathrm{H}, J 8.9 \mathrm{~Hz}, \mathrm{Ph}-\mathrm{H}$ of Bz), 7.62-7.12(m, 8H, Ph- $\left.H\right), 5.85\left(\mathrm{t}, 1 \mathrm{H}, J_{3,4} 9.4 \mathrm{~Hz}, \mathrm{H}-3\right)$, 5.05(dd, 1H, J J,3 $9.4 \mathrm{~Hz}, \mathrm{H}-2), 4.91\left(\mathrm{~d}, 1 \mathrm{H}, J_{1,2} 3.7 \mathrm{~Hz}, \mathrm{H}-1\right), 4.56,4.48\left(\mathrm{AB}_{\mathrm{q}}, 2 \mathrm{H},{ }^{2} \mathrm{~J} 11.7 \mathrm{~Hz}, \mathrm{PhCH}_{2}\right.$ ), $4.36\left(\mathrm{dd}, 1 \mathrm{H}, J_{6,6} \cdot 12.5 \mathrm{~Hz}, J_{5,6} 2.5 \mathrm{~Hz}, \mathrm{H}-6\right), 4.20\left(\mathrm{dd}, 1 \mathrm{H}, J_{5,6}, 4.2 \mathrm{~Hz}, \mathrm{H}-6\right), 4.04-3.95(\mathrm{~m}, 1 \mathrm{H}, \mathrm{H}-5), 3.76(\mathrm{t}$, $\left.1 \mathrm{H}, J_{4,5} 9.4 \mathrm{~Hz}, \mathrm{H}-4\right), 3.43\left(\mathrm{~s}, 3 \mathrm{H}, \mathrm{OCH}_{3}\right), 2.10,1.96\left(2 \mathrm{~s}, 6 \mathrm{H}, 2 \mathrm{COCH}_{3}\right) .6$ '. 7.38-7.30(m, 5H, Ph- $\left.\mathrm{H}\right), 5.08(\mathrm{t}$, $\left.J_{3,4} 8.4 \mathrm{~Hz}, \mathrm{H}-3\right), 5.06(\mathrm{~s}, 1 \mathrm{H}, \mathrm{H}-1), 4.86,4.60\left(\mathrm{AB}_{\mathbf{q}}, 2 \mathrm{H},{ }^{2} \mathrm{~J} 11.4 \mathrm{~Hz}, \mathrm{PhCH}_{2}\right), 4.76\left(\mathrm{~d}, 1 \mathrm{H}, J_{2,3} 7.6 \mathrm{~Hz}, \mathrm{H}-2\right)$, $4.34\left(\mathrm{dd}, 1 \mathrm{H}, J_{6,6}, 12.3 \mathrm{~Hz}, J_{5,6} 1.9 \mathrm{~Hz}, \mathrm{H}-6\right), 4.22\left(\mathrm{dd}, 1 \mathrm{H}, J_{5,6}, 4.8 \mathrm{~Hz}, \mathrm{H}-6\right), 3.88-3.79(\mathrm{~m}, 1 \mathrm{H}, \mathrm{H}-5), 3.59(\mathrm{dd}$, $\left.1 \mathrm{H}, J_{4,5} 9.5 \mathrm{~Hz}, \mathrm{H}-4\right), 3.40\left(\mathrm{~s}, 3 \mathrm{H}, \mathrm{OCH}_{3}\right) .2 .00\left(\mathrm{~s}, 3 \mathrm{H}, \mathrm{COCH}_{3}\right) .7$. $7.72(\mathrm{~d}, 2 \mathrm{H}, J 8.7 \mathrm{~Hz}, \mathrm{Ph}-H$ of Ts$), 7.52-$ $6.81(\mathrm{~m}, 12 \mathrm{H}, \mathrm{Ph}-H), 4.85\left(\mathrm{dd}, 1 \mathrm{H}, J_{2,3} 3.4 \mathrm{~Hz}, J_{3,4} 8.9 \mathrm{~Hz}, \mathrm{H}-3\right), 4.70-4.47(\mathrm{~m}, 5 \mathrm{H}, 2 \mathrm{PhCH}, \mathrm{H}-1), 4.40-$ $3.60(\mathrm{~m}, 5 \mathrm{H}, \mathrm{H}-2,4,5,6), 3.32\left(\mathrm{~s}, 3 \mathrm{H}, \mathrm{OC} H_{3}\right), 2.38\left(\mathrm{~s}, 3 \mathrm{H}, \mathrm{CH}_{3}\right), 2.03\left(\mathrm{~s}, 3 \mathrm{H}, \mathrm{COCH}_{3}\right) .8$ '. $8.10(\mathrm{~d}, 2 \mathrm{H}, J 6.8 \mathrm{~Hz}$, $\mathrm{Ph}-H$ of Bz), 7.60-7.28(m, 8H, $\mathrm{Ph}-H), 5.85-5.75\left(\mathrm{~m}, 1 \mathrm{H}, \mathrm{CH}_{2}=\mathrm{C} H\right), 5.50\left(\mathrm{dd}, 1 \mathrm{H}, J_{2,3} 3.3 \mathrm{~Hz}, \mathrm{H}-3\right), 5.22(\mathrm{~m}$, $\left.1 \mathrm{H}, J_{\text {trans }} 15.0 \mathrm{~Hz},{ }^{2} \mathrm{~J} 1.3 \mathrm{~Hz}, \mathrm{HCH}=\mathrm{CH}\right), 5.09\left(\mathrm{~m}, 1 \mathrm{H}, J_{\text {cis }} 8.3 \mathrm{~Hz}, H \mathrm{CH}=\mathrm{CH}\right), 4.76\left(\mathrm{~d}, 1 \mathrm{H}, J_{1,2} 0.5 \mathrm{~Hz}, \mathrm{H}-1\right)$, $4.73,4.56\left(\mathrm{AB}_{\mathrm{q}}, 2 \mathrm{H},{ }^{2} \mathrm{~J} 9.2 \mathrm{~Hz}, \mathrm{PhCH}_{2}\right), 4.35\left(\mathrm{~d}, 2 \mathrm{H}, J_{5,6} 2.7 \mathrm{~Hz}, \mathrm{H}-6\right), 4.05-4.12\left(\mathrm{~m}, 2 \mathrm{H}, \mathrm{CH}_{2}=\mathrm{CH}-\mathrm{CH}_{2}\right)$, $4.05\left(\mathrm{t}, 1 \mathrm{H}, J_{3,4} J_{4,5} 8.7 \mathrm{~Hz}, \mathrm{H}-4\right), 3.92\left(\mathrm{dd}, 1 \mathrm{H}, J_{2,3} 2.0 \mathrm{~Hz}, \mathrm{H}-2\right), 3.40\left(\mathrm{~s}, 3 \mathrm{H}, \mathrm{OCH} \mathrm{H}_{3}\right), 2.12(\mathrm{~s}, 3 \mathrm{H}, \mathrm{COCH})_{3}$. $"$ $8.11(\mathrm{~d}, 2 \mathrm{H}, J 8.4 \mathrm{~Hz}, \mathrm{Ph}-H$ of Bz), 7.66-7.12(m, 8H, Ph- $H), 6.15\left(\mathrm{~d}, 1 \mathrm{H}, J_{1,2} 1.8 \mathrm{~Hz}, \mathrm{H}-1\right), 5.85-5.75(\mathrm{~m}, 1 \mathrm{H}$, $\left.\mathrm{CH}_{2}=\mathrm{CH}\right), 5.51\left(\mathrm{dd}, J_{2,3} 4.2 \mathrm{~Hz}, \mathrm{H}-3\right), 5.20\left(\mathrm{~m}, 1 \mathrm{H}, J_{\text {trans }} 16.3 \mathrm{~Hz},{ }^{2} \mathrm{~J} 0.9 \mathrm{~Hz}, \mathrm{HC} H=\mathrm{CH}\right), 5.10\left(\mathrm{~m}, 1 \mathrm{H}, J_{\text {cis }} 9.8\right.$ $\mathrm{Hz}, H \mathrm{CH}=\mathrm{CH}), 4.74,4.60\left(\mathrm{AB}_{\mathrm{q}}, 2 \mathrm{H},{ }^{2} \mathrm{~J} 11.6 \mathrm{~Hz}, \mathrm{PhCH}_{2}\right), 4.12\left(\mathrm{t}, 1 \mathrm{H}, J_{3,4} J_{4,5} 9.3 \mathrm{~Hz}, \mathrm{H}-4\right), 4.12-4.00(\mathrm{~m}, 3 \mathrm{H}$, $\left.\mathrm{H}-5, \mathrm{CH}_{2}=\mathrm{CH}-\mathrm{CH}_{2}\right), 3.91\left(\mathrm{dd}, 1 \mathrm{H}, J_{2,3} 0.6 \mathrm{~Hz}, \mathrm{H}-2\right), 2.14,2.08\left(2 \mathrm{~s}, 6 \mathrm{H}, \mathrm{COCH}_{3}\right)$.

(Received in China 15 April 1997; revised 29 May 1997; accepted 1 July 1997) 